\title{
Suppression of cavitation-induced nucleation in systems under isochoric confinement
}

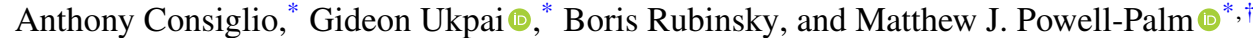 \\ Department of Mechanical Engineering, University of California at Berkeley, Berkeley, California 94720, USA
}

(Received 9 March 2020; accepted 1 June 2020; published 16 June 2020)

\begin{abstract}
Stabilization of supercooled liquids presents a fundamental challenge with broad practical implications. Here, we unify divergent aspects of bubble dynamics, thermodynamics, and nucleation theory to explore the influence of confinement on cavitation-induced nucleation, a principal destabilization mechanism in supercooled systems. We demonstrate that confinement can suppress cavity collapse even in remarkably large systems and reveal the existence of critical volumes at which cavitation-induced nucleation becomes entirely kinetically prohibited, suggesting confinement as a compelling route towards modulation of kinetic phase change processes.
\end{abstract}

DOI: 10.1103/PhysRevResearch.2.023350

\section{INTRODUCTION}

Efforts to mediate the nucleation of solid phases in supercooled liquids are ubiquitous in fields ranging from materials science [1] to food science [2], and nucleation suppression has recently emerged as an essential route towards long-term organ and tissue preservation [3-5]. Nucleation is difficult to avoid in mobile or industrial contexts however [6], because acoustic agitations of any kind can cause the liquid phase to cavitate, resulting in ultrarapid, high-pressure nucleation events $[7,8]$. Here, we explore the effects of isochoric (constant-volume) confinement on the cavitation dynamics and nucleation kinetics of aqueous systems exposed to ultrasonication, one of most reliable sources of rapid nucleation [9]. We unify bubble dynamics, thermodynamics, and classical nucleation theory to demonstrate that confinement across multiple volume scales can dramatically decrease peak cavity collapse pressures and dampen cavitation-induced nucleation under a wide range of operating conditions. Furthermore, we demonstrate the existence of a critical confinement volume, on the order of $10^{8}$ times larger than the cavitating bubble itself, beneath which cavitation-induced nucleation becomes entirely kinetically prohibited. Our results reveal fundamental insights into the effects of confinement on kinetic phase change processes, and suggest that confinement could provide a compelling route towards high-stability supercooling.

Mechanical and acoustic stimuli have long been known to induce ice nucleation [10], and the works of Hickling [7,8] clarified the mechanism to be the collapse of cavitating bubbles within the liquid. Collapse events occur over the span

\footnotetext{
*These authors contributed equally to this work.

${ }^{\dagger}$ Author to whom correspondence should be addressed: mpowellp@berkeley.edu

Published by the American Physical Society under the terms of the Creative Commons Attribution 4.0 International license. Further distribution of this work must maintain attribution to the author(s) and the published article's title, journal citation, and DOI.
}

of nanoseconds, and can cause local pressure excursions on the order of several gigapascals in the surrounding medium. In low-thermal-diffusivity media such as water, this dynamic compression is sufficiently rapid to be considered quasiisentropic [7], and will result in liquid water rapidly traversing the equilibrium domains of several high-pressure ice polymorphs. Ice VII clusters will form nigh instantaneously during such dynamic compression processes [8,11,12], and it is assumed that these short-lived clusters then serve as ultrapotent nucleation sites for ice-1h upon return of the water to its initial temperature and pressure [7], producing the macroscopic result of rapid ice-1h formation associated with sonic agitation of supercooled water [9].

Given the extreme consistency of cavitation-induced nucleation, ultrasonicated systems (which aggressively cavitate) provide an excellent platform for the study of nucleation suppression. In our recent experimental work [13], we observed that macroscopic volumes of supercooled water $(\sim 100 \mathrm{~mL})$ confined in an isochoric container were resistant to nucleation via ultrasonication as well as other mechanoacoustic stimuli, suggesting the absence or dampening of cavitation-induced nucleation processes. In the present work, we conduct a fundamental analysis of the effects of multiscale confinement on cavitation-induced ice nucleation, and in the process uncover fundamental limiting behaviors relevant to cavity collapse, nucleation, and the broader spectrum of kinetic processes under confinement.

\section{CAVITATION DYNAMICS IN CONFINED SYSTEMS}

In formulating this analysis, we must first acknowledge that the nucleation phenomena of interest are mathematically linked to cavitation via quasi-isentropic compression, the magnitude of which is dependent upon the pressure excursions encountered during cavity collapse. Thus, we begin by analyzing the dependence of cavity collapse dynamics on confinement.

We develop a modified spherical finite-domain Gilmore model [14] to describe the collapse dynamics of a single spherical air bubble of initial radius $R_{0}$ within a confined spherical body of water of radius $R_{c}$ exposed to ultrasonic 


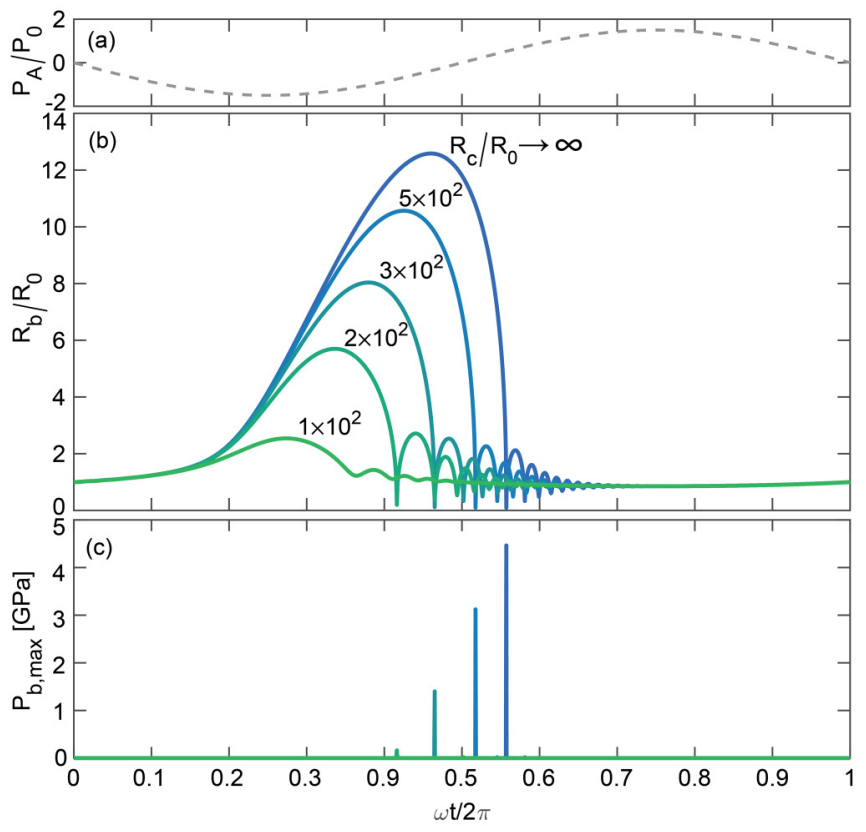

FIG. 1. Transient excitation of a gas bubble in $0{ }^{\circ} \mathrm{C}$ water under an ultrasonic pressure field. (a) Acoustic pressure signal of frequency $40 \mathrm{kHz}$ and amplitude 1.5 bar as a function of cycle period, $\omega t / 2 \pi$. (b) Time evolution of relative bubble radius, $R_{b} / R_{0}$, for various values of relative confinement radius, $R_{c} / R_{0}$. Equilibrium bubble radius, $R_{0}=2.09 \mu \mathrm{m}$. (c) Pressure of water at bubble interface.

stimulation. These dynamics are governed by an equation of the form

$$
\begin{aligned}
& {\left[-r\left(1-\frac{u}{c}\right) \frac{D u}{D t}-\frac{3}{2}\left(1-\frac{u}{3 c}\right) u^{2}+\frac{r c}{K}\left(1-\frac{u}{c}\right) \frac{D p}{D t}\right.} \\
& \left.+\left(1+\frac{u}{c}\right) \int_{p_{0}}^{p} \frac{d p}{\rho}\right]_{R_{b}}^{R_{c}}=0,
\end{aligned}
$$

which describes the motion of the liquid phase between the boundary of the cavitating bubble and the confined container, and reduces to the classical Gilmore equation in the limit of infinite container volume. Here, $r$ is the radial coordinate, $u$ is the radial velocity, $p$ is the pressure, $c$ is the speed of sound in the liquid, $\rho$ is the density of the liquid, $K$ is the bulk modulus of the liquid, and $R_{b}$ is the transient bubble radius. The material properties of water are evaluated at the initial bulk temperature of the system, according to the multiparameter equation of state maintained by the International Association for the Properties of Water and Steam (IAPWS) [15]. A detailed account of the full model and derivation is available in Appendix A.

The resulting bubble growth and collapse dynamics are depicted in Fig. 1 for varying confinement volumes. The bubble initially grows as tension is applied by the pressure field [shown in Fig. 1(a)], reaching a maximum size following the point of minimum applied pressure. As the applied tension is then released, a force imbalance at the interface of the oversized bubble causes violent collapse [Fig. 1(b)], during which the bubble radius may recoil to less than one tenth its equilibrium size. Given that the pressure within the bubble varies as $R_{b}{ }^{-3 \gamma}$ (in which $\gamma$ is the ratio of specific heats for air), these collapse events result in brief periods ( $0.5-2 \mathrm{~ns})$ of extreme pressure, as shown in Fig. 1(c).

The degree to which the system is confined (i.e., the ratio of the total confined system volume to the initial bubble volume) significantly alters bubble dynamics, acutely reducing the maximum bubble size reached and the peak pressure experienced during collapse [Figs. 1(b) and 1(c)]. Physically speaking, this reduction is driven by the finite compressibility of the confined liquid phase; as the bubble grows, the reduction of the volume occupied by water must be accompanied by an increase in hydrostatic pressure, which retards further growth of the bubble.

In Fig. 2, the according effects of confinement on peak collapse pressure are demonstrated for varying system conditions, across which three distinct behavior regimes emerge [Fig. 2(a)]. At the limit of infinite system volume, which is here approached at system volumes on the order of $10^{11}$ times greater than the initial bubble volume, the bubble dynamics are unaffected by the container. We term this volume scale the isobaric limit, as the results become equivalent to a system operating under unconfined isobaric conditions. It should be noted that this limit may increase somewhat (e.g., the effects of confinement may be felt at greater system volumes) as the initial bubble size is decreased, due to a relative enhancement of surface tension effects (See Appendix B for more detail).

As the degree of confinement increases (i.e., the container volume decreases), the bubble dynamics enter a "transition zone", in which the bubble begins to feel the effect of confinement and the peak collapse pressures begin to decline. The degree of confinement at which the transition zone is centered is dominated by the compressibility of the liquid phase, while the width of the transition zone is a weak function of the interfacial surface tension of the liquid, the ratio of specific heats of the gas, and the viscosity of the liquid. Neglecting time-dependent effects, the position of the transition zone can be probed by performing a simple force balance (derived in Appendix B) at the bubble's maximum size, yielding the scaling relation:

$$
\begin{aligned}
K & \ln \left(\frac{R_{c}^{3}-R_{b, 0}^{3}}{R_{c}^{3}-R_{b, \max }^{3}}\right)+p_{l, 0} \\
& =\left(p_{l, 0}+\frac{2 \sigma}{R_{b, 0}}\right)\left(\frac{R_{b, 0}}{R_{b, \max }}\right)^{3 \gamma}-\frac{2 \sigma}{R_{b, \max }}+P_{A},
\end{aligned}
$$

in which $K$ is the bulk modulus of the liquid, $\sigma$ is the surface tension, $p_{l, 0}$ is the initial pressure (atmospheric), $P_{A}$ is a driving pressure, $\gamma$ is the ratio of specific heats of the air, and $R_{0}, R_{b, \max }$, and $R_{c}$ are the initial bubble radius, maximum bubble radius, and confinement radius, respectively.

This scaling relation describes the approximate dependence of the maximum bubble radius on confinement, which serves as an effective first-order proxy for the peak collapse pressure and allows for convenient approximation of the order-of-magnitude confinement scales at which an arbitrary liquid with bulk modulus $K$ and surface tension $\sigma$ will start to see an arrest of cavitation dynamics. These scales are plotted for varying bulk moduli and surface tensions in Appendix B, and the location and width of the water transition zone 

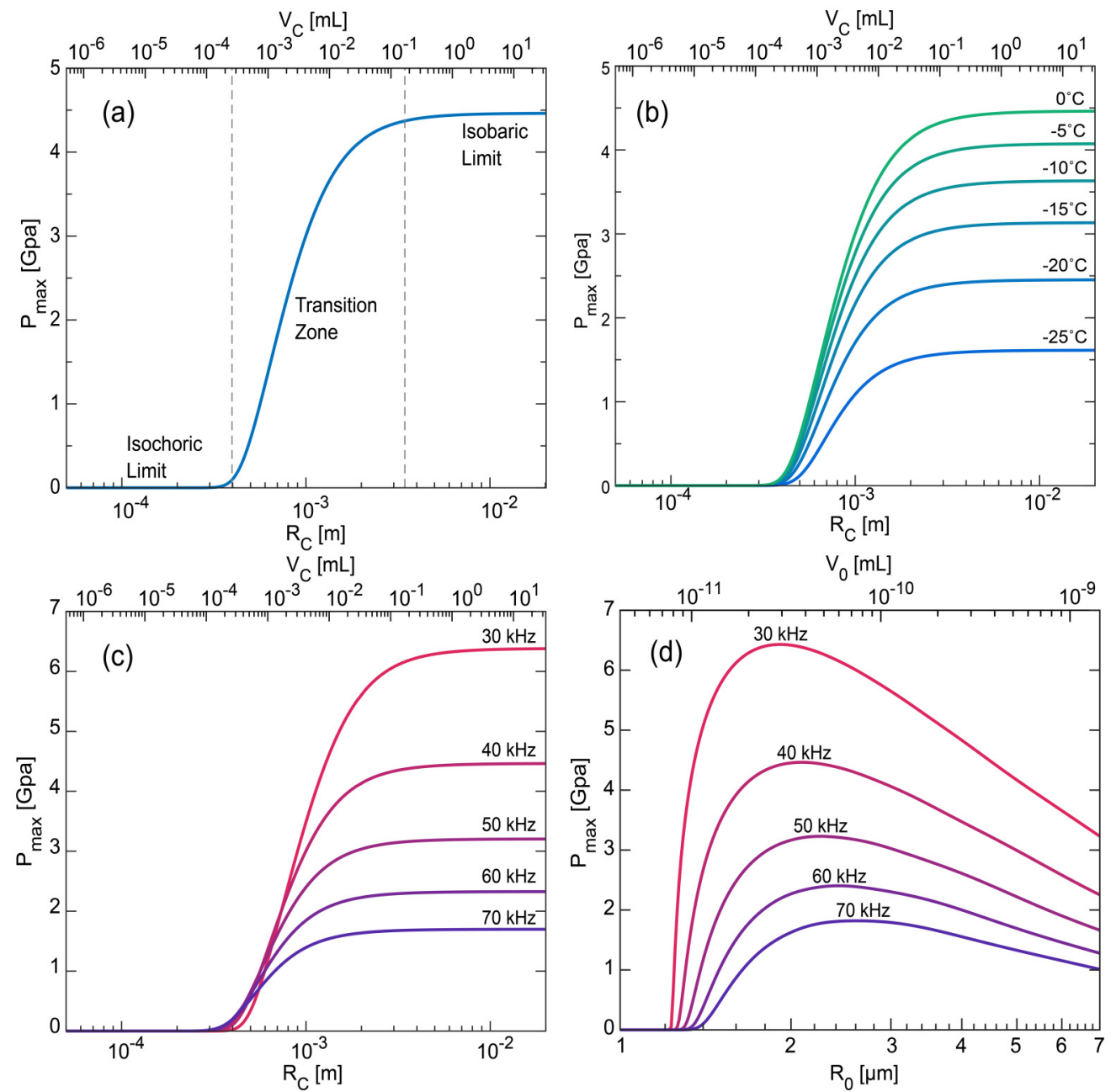

FIG. 2. Peak collapse pressures experienced during cavitation in confined volumes. Curves are plotted for an excitation frequency of $40 \mathrm{kHz}$ unless otherwise noted. (a) General dependence of maximum cavitation pressure on degree of system confinement. (b) Dependence of maximum cavitation pressure on degree of system confinement for varying degrees of bulk supercooling. (c) Dependence of maximum cavitation pressure on excitation frequency. (d) Dependence of maximum cavitation pressure on initial bubble radius at the isobaric limit of system confinement.

predicted by this simple relation demonstrate order-ofmagnitude agreement with the full data presented in Fig. 2.

The third and final regime depicted in Fig. 2(a), encountered at system volumes approximately $10^{7}$ times larger than the initial bubble volume, demonstrates the existence of a critical confinement under which bubble collapse becomes entirely prohibited (as evidenced by the peak collapse pressure reducing to zero). Resistance from the rigid container prevents the bubble from reaching sufficient size to drive an unstable collapse event, and it instead merely oscillates stably in response to the applied pressure field [as in the bottommost profile in Fig. 1(b)]. We term this volume range the isochoric limit, and at confinement volumes in this regime, the system will experience no significant excursions in pressure.

The three regimes identified in Fig. 2(a) remain consistent as operational parameters are varied, though the discrete peak pressures encountered may change significantly. In particular, reduction of the bulk system temperature significantly dampens collapse intensity [Fig. 2(b)], due principally to the large increase in the viscosity of water at subzero centigrade tem- peratures, and reduction of the excitation frequency [Fig. 2(c)] increases collapse intensity, due to an increased tensioning period in which the bubble is allowed to grow preceding collapse. The dependence of peak collapse pressure on the initial bubble size is also shown in Fig. 2(d), acknowledging that in an experimental context, a bubble field will exist with a distribution of cavitating bubble sizes. Throughout the collapse analyses presented in this work, the initial bubble radius that yields the highest collapse pressure for a given frequency is employed, ensuring description of the most extreme possible response.

\section{TRANSIENT SUPERCOOLING DURING CAVITY COLLAPSE}

Using the results of Fig. 2, we are now empowered to relate confinement to high-pressure transient ice nucleation by evaluating the quasi-isentropic thermodynamic path taken by the water surrounding the bubble during collapse. In Fig. 3(a), compression isentropes for water evaluated from multiple 

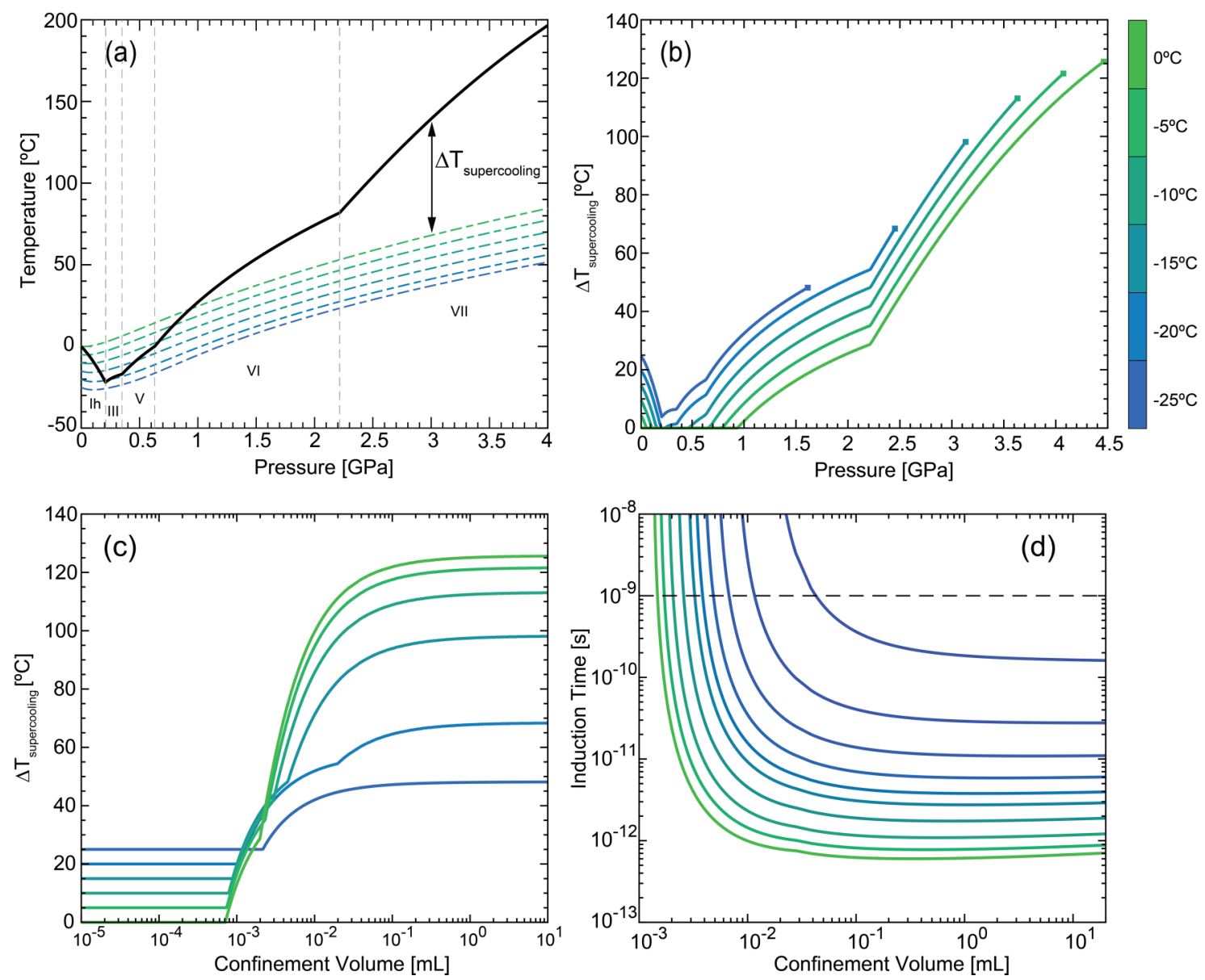

FIG. 3. Transient high-pressure solidification processes for systems of varying bulk temperatures sonicated at $40 \mathrm{kHz}$. (a) Phase diagram of water with isentropic compression curves for bulk system temperatures of $0,-5,-10,-15,-20$ and $-25^{\circ} \mathrm{C}$, color coded according to the color bar at right. The water-ice liquidus line (solid black) shows the equilibrium boundary between the liquid water phase (above the line) and the ice phases Ih, III, V, VI, and VII (below the line), as labeled along the bottom of the plot. Isentropic compression curves (dashed lines) show the temperature-pressure thermodynamic path followed by water as it is isentropically compressed from varying initial temperatures. As compression increases, the water traverses through the equilibrium regions of several different ice phases. Both the liquidus line and the isentropic compression curves are calculated using the standard IAPWS multiparameter equations of state [15,16]. (b) Magnitude of transient supercooling encountered during isentropic compression, e.g., the difference $\Delta T_{\text {supercooling }}$ between the liquidus curve and a given isentropic compression curve. Curves are truncated at the maximum pressure reached during cavity collapse for a given bulk temperature. (c) The same maximum transient supercooling as a function of confinement volume, adapted according to the pressure-confinement volume relations provided in Fig. 2. (d) Minimum ice VII induction time encountered during cavity collapse as a function of confinement volume, as calculated using classical nucleation theory. The dashed line represents the critical induction time threshold, defined by the average duration of a high-pressure collapse event (here $1 \mathrm{~ns}$ ). Minimum ice VII induction times beneath this threshold will lead to formation of ice VII nuclei during cavity collapse, while induction times above the threshold will not.

bulk system temperatures are plotted atop the $\mathrm{H}_{2} \mathrm{O}$ phase diagram, allowing calculation of the temperature difference

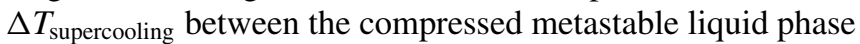
and the relevant equilibrium phase of ice as a function of pressure. This temperature difference is plotted in Fig. 3(b) for various isentropes. As the water is compressed, it can pass through the equilibrium regions of up to five high pressure polymorphs of ice, with the most radical supercooling invariably occurring in the ice VII region.

By now combining the confinement-pressure results of Fig. 2(b) with the pressure-supercooling results of Fig. 3(b), the maximum transient supercooling encountered at a given confinement volume can be calculated [Fig. 3(c)], and the same confinement limits seen in the collapse pressure can be observed. At the isochoric limit, the maximum supercooling encountered in the system will simply be defined by the bulk system temperature, as no cavitation will occur. At the isobaric limit, significant cavity collapse pressures will drive $\Delta T_{\text {supercooling }}$ into the range of hundreds of degrees, principally in the ice VII region of the phase diagram.

Reduction of the maximum transient supercooling can be observed at remarkably large confinement volumes, on the order of $10^{9}$ times larger than the bubble itself, and increases acutely over the volume range corresponding to the transition zone. Across confinement volumes, the maximum supercooling encountered is also a strong function of the bulk system 
temperature, though counterintuitively, increasing bulk supercooling decreases later cavitation-induced supercooling, due to the increasing viscosity of water at low temperatures.

\section{EFFECTS ON CAVITATION-INDUCED NUCLEATION}

We now incorporate the transient supercooling results developed in Fig. 3(c) into classical nucleation theory (CNT) and examine the induction time required for the nucleation of a high-pressure solid phase as a function of confinement. To simplify this analysis, we make the crucial assumption that the only high-pressure polymorph of ice likely to form during the quasi-isentropic compression process is ice VII, though each isentrope will briefly pass through regions in which ice V or ice VI may be more thermodynamically stable. This assumption is based on previous experimental observations of ice nucleation during quasi-isentropic dynamic shock compression, in which metastable ice VII was found to form preferentially to stable ice VI due its lower interfacial free energy $[17,18]$. Furthermore, growth of ice VII has been observed at the same time scales as cavity collapse ( $\sim$ single nanoseconds) $[12,18,19]$, and the relative degree of supercooling is highest in the ice VII region.

Using the Myint equation of state [20] to calculate the thermodynamic driving forces (e.g., the chemical potential difference) between compressed water and ice VII along each compression isentrope, CNT enables calculation of a transient induction time of the form

$$
\tau=\frac{8 k_{B} T}{\pi^{2} \lambda D^{*}},
$$

which was originally formulated by Kaschiev [21] and adapted by Myint [11] to describe the time required for a cluster of ice VII particles to reach the critical size required for nucleation. In Eq. (3), $k_{B} T$ is the energy scaling factor, $D^{*}$ is a frequency factor describing the rate of attachment of additional molecules to a critical cluster, and $\lambda$ describes the curvature at the top of the energy barrier (see Appendix C for a full accounting of nucleation parameters).

Armed with the maximum supercooling encountered at a given confinement volume [Fig. 3(c)] and the pressuretemperature paths followed during quasi-isentropic compression of the water, the fastest induction time possible at a given confinement volume can be calculated for various degrees of bulk supercooling [Fig. 3(d)]. The isobaric and isochoric limiting behaviors are once again observable: the induction time stabilizes to a minimum value at large system volumes, and it arcs toward infinity as the system volume decreases and the cavitation dynamics driving nucleation are extinguished.

In order to estimate whether cavitation-induced ice VII nucleation will ultimately occur, we can now compare the induction time required for the formation of a stable nucleus to the period over which the pressure excursion due to cavity collapse occurs. Previous experimental work has observed ultrarapid formation of metastable ice VII at dynamic pressures as low as $1.8 \mathrm{GPa}$ [17]; we thus examined the period over which the collapse pressure remained greater than or equal to this pressure, finding across bulk system temperature and frequency conditions a value of approximately $0.5-1.5 \mathrm{~ns}$. This period can be applied as a critical threshold for the

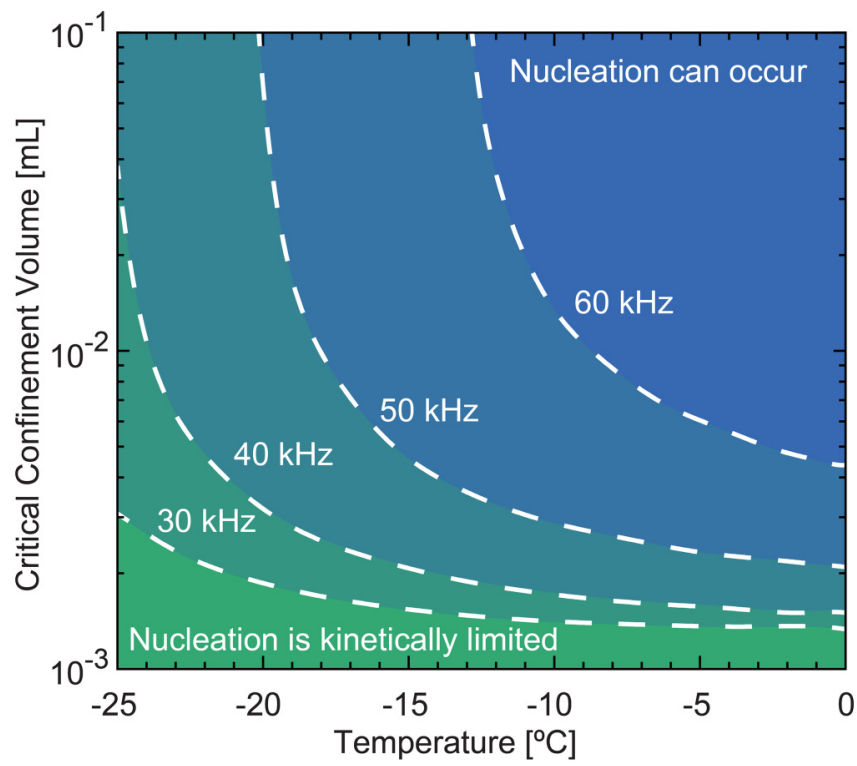

FIG. 4. Ice VII kinetic phase diagram. The critical confinement volume for kinetic prohibition of ice VII nucleation is plotted as a function of temperature for various driving frequencies. Dashed lines indicate the boundary that separates the confinement regimes in which nucleation of ice VII can (above a given line) and cannot (beneath a given line) occur.

induction time of a high-pressure solid phase, and is plotted as a dashed line in Fig. 3(d). If the calculated induction time at a given confinement volume rests under this threshold, ice VII has a kinetic route to nucleation. If the induction time is above this threshold however (i.e., if it takes longer than $\sim 1 \mathrm{~ns}$ for a critical cluster of ice VII to form), the collapse pressure will dissipate before a stable nucleus can form, kinetically prohibiting cavitation-induced nucleation.

The discrete confinement volumes at which the induction time curves plotted in Fig. 3(d) cross the collapse period threshold thus represent a critical phenomenon: the degree of confinement at which cavitation-induced nucleation becomes kinetically prohibited. This "critical confinement" volume can be plotted as a function of system parameters, herein the bulk temperature of the resting system and the driving frequency of the ultrasonication, to develop a kinetic phase diagram establishing the temperature-confinement boundary under which cavitation-induced nucleation will not occur for a given driving frequency (Fig. 4).

The single-bubble analysis performed herein reveals several important consequences of system confinement: it can dampen bubble collapse dynamics, reduce transient supercooling during cavitation, and ultimately restrict cavitationinduced nucleation. Furthermore, there exist calculable critical confinement volumes at which discrete kinetic behaviors (bubble collapse, nucleation of high-pressure polymorphs) can be prohibited entirely. Perhaps most surprising, however, is the volume scale at which these myriad confinement effects come into play: For a single cavitation bubble on the order of $10^{-11} \mathrm{~mL}$ in volume, across sonication frequencies and bulk system temperatures, bubble collapse and cavitation-induced nucleation become kinetically prohibited at minimum critical 
confinement volumes on the order of $10^{-4} \mathrm{~mL}$ and $10^{-3} \mathrm{~mL}$, respectively. From a physical perspective, this remarkable difference between the requisite system volumes for critical confinement and the cavitating bubble volumes is a manifestation of the large compressibility difference between water and air (or more generally between generic liquids and gases); if the compressibility of the liquid medium surrounding the bubble increases, the observed difference in these volume scales lessens, consistent with the scaling relation provided in Eq. (2) and plotted in Appendix B in Fig. 6.

\section{DISCUSSION}

These remarkably large single-bubble confinement volumes suggest that the observed confinement effects may also translate to bulk macroscopic systems, which produce large populations of simultaneously cavitating bubbles upon ultrasonication. For example, recent estimations of the number density of cavitating bubbles in water sonicated in the 20 $200 \mathrm{kHz}$ frequency range suggest that approximately $10^{3}$ bubbles may cavitate per $\mathrm{mL}$ of liquid [22]. As a first-order approximation, if a hypothetical bulk system is ultrasonicated at $30 \mathrm{kHz}$ and confined at $10 \mathrm{~mL}$, the volume may be divided evenly by the number density of bubbles to arrive at an effective confinement volume per bubble of $10^{-2} \mathrm{~mL}$, which is well within the transition zone for transient supercooling [Fig. 3(c)] and approaching the critical confinement threshold for cavitation-induced ice VII nucleation (Fig. 4).

It must be noted, however, that the critical confinement volumes calculated in this work describe only the most extreme possible cavitation scenario, in which the equilibrium size of the cavitating bubble corresponds to the peak displayed in Fig. 2(d), which will yield the greatest possible collapse pressure and the highest pursuant likelihood of ice VII nucleation. Experimentally, a cavitating bubble field in a macroscopic system will include a wide distribution of larger and smaller bubbles [22], which will inevitably produce significantly smaller pressure excursions upon collapse and reduce the likelihood of nucleation. Nucleation-suppressing confinement effects may thus potentially be observed at much larger scales than those considered here, and indeed in our recent proof-of-concept experimental investigation of nucleation in supercooled confined systems [13], suppression of nucleation was observed in macroscopically confined systems on the order of $100 \mathrm{~mL}$ that were supercooled to $-3{ }^{\circ} \mathrm{C}$ and ultrasonicated at $55 \mathrm{kHz}$. Although a full bubble field analysis is required to accurately extend the present model to experimentally relevant multibubble systems, the experimental findings described in Ref. [13] provide preliminary experimental confirmation of our proposed nucleation suppression mechanism, and future experimental efforts should not rule out confinement as a means of suppressing cavitationinduced effects at any scale. It should also be noted that the bubble number density, size distributions, and single-bubble peak collapse pressures may vary significantly for acoustic agitations other than ultrasonication, and future work should adapt the analyses presented herein to a variety of acoustic stimuli.

The need for robust control of ice nucleation has become increasingly clear in light of recent successes in medical supercooling [3,4], which have yielded excellent biological results but have thus far not proven translatable outside a highly controlled laboratory environment [6]. Cavitation-induced nucleation of high-pressure polymorphs is the dominant mechanism by which supercooled systems are destabilized upon mechanical or acoustic perturbation (as occurs variously during transportation and clinical use), and thus any supercooling approach intended for practical use must work to suppress this mechanism.

Our results herein suggest that isochoric confinement can significantly hinder cavitation-induced nucleation, and, taken in combination with other recent works investigating the limiting effects of isochoric confinement on other ice nucleation and growth mechanisms $[13,23,24]$, suggest that confinement may provide a compelling route toward robust nucleation suppression in supercooled systems. Future work should extend the single-bubble analyses developed herein to full cavitating bubble fields, and in doing so establish a model for direct prediction of nucleation suppression effects in more complex macroscale cavitating systems.

\section{ACKNOWLEDGMENTS}

The authors thank Professor S. Morris for several helpful conversations. The authors acknowledge no external funding for this work.

\section{APPENDIX A: DERIVATION OF THE CAVITATION MODEL}

Here we present the details of the mathematical model developed in the study to model bubble dynamics. The derivations presented follow the approach implemented by Gilmore [14], modified for consideration of a finite domain. The problem considered herein describes cavitation dynamics under confinement in an isochoric container subjected to an ultrasonic pressure field, enabling study of collapse effects as a function of container size. The mathematical formulation is defined by a single spherical bubble located at the center of a rigid, spherical vessel, as shown in Fig. 5.

Radial, compressible flow is prescribed, and the gas inside the bubble is assumed to be spatially uniform. The effect of gravity and any initial temperature variations are neglected. Thus, the equations of motion describing the conservation of mass and momentum in the liquid are

$$
\begin{aligned}
& \frac{1}{\rho} \frac{D \rho}{D t}+\frac{1}{r^{2}} \frac{\partial\left(r^{2} u\right)}{\partial r}=0, \\
& \frac{\partial u}{\partial t}+u \frac{\partial u}{\partial r}+\frac{1}{\rho} \frac{\partial p}{\partial r}=0,
\end{aligned}
$$

where $\rho$ is the density, $u$ is the velocity and $p$ is the pressure. The viscosity enters the problem solely as a boundary condition, as will be seen later, since viscous effects are confined to a thin boundary layer at the bubble surface and have been found to have a negligible effect on bubble dynamics $[14,25,26]$.

The momentum equation is integrated from the liquid-gas interface at the bubble wall, $R_{b}$, to the container wall or 


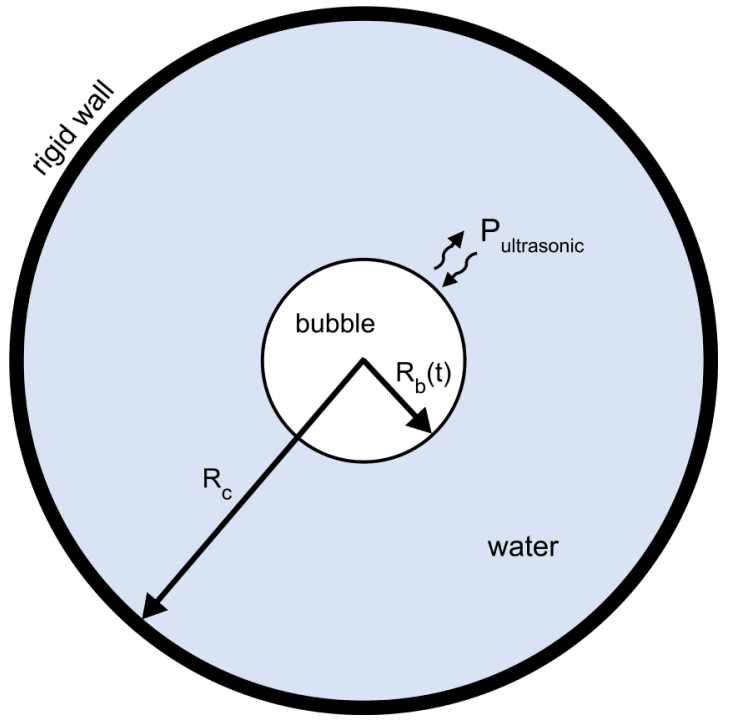

FIG. 5. Schematic of spherical bubble within a rigid confinement

confinement radius, $R_{c}$ :

$\int_{R_{b}}^{R_{c}}\left[\frac{\partial u}{\partial t}+u \frac{\partial u}{\partial r}+\frac{1}{\rho} \frac{\partial p}{\partial r}\right] d r=\int_{R_{b}}^{R_{c}} \frac{\partial u}{\partial t} d r+\left[\frac{1}{2} u^{2}+h\right]_{R_{b}}^{R_{c}}$,

where the enthalpy at constant entropy, $h$, is given as

$$
h=\int_{p_{0}}^{p} \frac{d p}{\rho} .
$$

Here we use the definitions

$$
\begin{gathered}
\frac{1}{r^{2}} \frac{\partial\left(r^{2} u\right)}{\partial t}=\frac{\partial u}{\partial t}+\frac{2 u}{r}=\Delta_{l}, \\
\frac{D u}{D t}=\frac{\partial u}{\partial t}+u \frac{\partial u}{\partial r, b} .
\end{gathered}
$$

The remaining integral in Eq. (A3) can be reformulated by partial integration to obtain

$$
\begin{aligned}
\int_{R_{b}}^{R_{c}} \frac{\partial u}{\partial t} d r & =\left[-r \frac{\partial u}{\partial t}\right]_{R_{b}}^{R_{c}}+\int_{R_{b}}^{R_{c}} r \frac{\partial \Delta_{l}}{\partial t} d r \\
& =\left[-r \frac{D u}{D t}+r u \Delta_{l}-2 u^{2}\right]_{R_{b}}^{R_{c}}+\int_{R_{b}}^{R_{c}} r \frac{\partial \Delta_{l}}{\partial t} d r .
\end{aligned}
$$

The remaining integral in Eq. (A7) can be evaluated by recognizing that the quantity $r \Delta_{l}$ is invariant in the acoustic approximation and thus satisfies the relation

$$
\frac{D\left(r \Delta_{l}\right)}{D t}=\frac{\partial\left(r \Delta_{l}\right)}{\partial t}+c \frac{\partial\left(r \Delta_{l}\right)}{\partial r}=0
$$

where $c$ is the speed of sound in the liquid. Utilizing this, the integrated momentum equation can be assembled to yield

$$
\left[-r \frac{D u}{D t}+r \Delta_{l}(u-c)-\frac{3}{2} u^{2}+h\right]_{R_{b}}^{R_{c}}=0 .
$$

From the continuity equation we find

$$
\Delta_{l}=-\frac{1}{\rho} \frac{D \rho}{D t}=-\frac{1}{K} \frac{D p}{D t} .
$$

In the barotropic approximation, pressure is only a function of density, $p=p(\rho)$, and is described by the relation

$$
K=\rho c^{2}=\rho \frac{d p}{d \rho},
$$

where $K$ is the liquid bulk modulus and $c$ is the speed of sound. Herein, $K$ and $c$ are evaluated at equilibrium conditions. Substituting this into Eq. (A9), we get

$$
\left[-r \frac{D u}{D t}+\frac{r c}{K}\left(1-\frac{u}{c}\right) \frac{D p}{D t}-\frac{3}{2} u^{2}+h\right]_{R_{b}}^{R_{c}}=0 .
$$

Gilmore [14] recognized that the Kirkwood-Bethe approximation, which assumes that the characteristic invariants propagate at a velocity of $(c+u)$, is more accurate for finite fluid velocities. By following the approach implemented by Flynn [25], we may compare the form of Gilmore's equation to Eq. (A12) and include three additional correction factors to yield

$$
\begin{aligned}
& {\left[-r\left(1-\frac{u}{c}\right) \frac{D u}{D t}-\frac{3}{2}\left(1-\frac{u}{3 c}\right) u^{2}+\frac{r c}{K}\left(1-\frac{u}{c}\right) \frac{D p}{D t}\right.} \\
& \left.\quad+\left(1+\frac{u}{c}\right) h\right]_{R_{b}}^{R_{c}}=0 .
\end{aligned}
$$

The enthalpy, $h$, as defined in Eq. (A4), is evaluated in terms of pressure

$$
h=\int_{p_{0}}^{p} \frac{d p}{\rho}=\frac{K}{\rho_{0}}\left(1-\exp \left\{\frac{p_{0}-p}{K}\right\}\right) .
$$

To obtain an equation describing the motion of the bubble interface, Eqs. (A13) and (A14) must be evaluated at the bubble wall and container wall. The boundary conditions satisfied by the liquid at the bubble wall, assuming no diffusion of gas through the interface, are

$$
\begin{gathered}
u\left(R_{b}, t\right)=\dot{R}_{b}, \\
p\left(R_{b}, t\right)=P_{g}(t)-\frac{2 \sigma}{R_{b}}-4 \mu \frac{\dot{R}_{b}}{R_{b}}+P_{A} \sin (\omega t),
\end{gathered}
$$

where, $R_{b}$ is the evolving bubble radius, $\sigma$ is the surface tension of the gas-liquid interface, $\mu$ is the liquid viscosity, $P_{g}(t)$ is the pressure of the gas within the bubble, $P_{A}$ is the amplitude and $\omega$ is the frequency of the imposed ultrasonic pressure disturbance. The overdot indicates $d / d t$.

Adopting a polytropic equation of state, the pressure within the bubble under adiabatic conditions is given by the relation

$$
P_{g}(t)=\left(p_{l, 0}+\frac{2 \sigma}{R_{b, 0}}\right)\left(\frac{R_{b, 0}}{R_{b}}\right)^{3 \gamma},
$$

where $\gamma$ is the ratio of specific heats, $c_{p} / c_{v}$. Since the container walls are assumed rigid, the liquid satisfies the condition

$$
u\left(R_{c}, t\right)=0
$$


It has been shown that large pressure variations due to bubble collapse during transient cavitation are only felt within a distance of few bubble radii from the bubble wall [7]. Thus, to a first-order approximation, it can be assumed that the density in the liquid varies uniformly due to the evolving bubble volume. Utilizing the relation from Eq. (A11), the pressure of the liquid at the container wall can written as

$$
p\left(R_{c}, t\right)=K \ln \left(\frac{R_{c}{ }^{3}-R_{b, 0}{ }^{3}}{R_{c}{ }^{3}-R_{b}{ }^{3}}\right)+p_{l, 0},
$$

where $R_{b, 0}$ is the initial bubble radius and $p_{l, 0}$ is the initial liquid pressure.

By evaluating Eqs. (A13) and (A14) utilizing the boundary conditions, a second-order nonlinear ordinary differential equation is obtained describing the time evolution of the bubble radius:

$$
\begin{aligned}
& \left(1-\frac{\dot{R}_{b}}{c}\right) R_{b} \ddot{R}_{b}+\frac{3}{2}\left(1-\frac{\dot{R}_{b}}{c}\right) \dot{R}_{b}^{2}+\frac{R_{c} c}{K} \frac{D P_{c}}{D t} \\
& -\frac{R_{b} c}{K} \frac{D P_{b}}{D t}\left(1-\frac{\dot{R}_{b}}{c}\right)+h_{c}-\left(1+\frac{\dot{R}_{b}}{c}\right) h_{b}=0 .
\end{aligned}
$$

This equation can be readily numerically integrated to find the bubble radius as a function of time, $R_{b}(t)$. It is easily observed that in the limit of infinite confinement radius, the model reverts to the traditional Gilmore equation describing bubble dynamics in an infinite (isobaric) medium.

In the analysis described herein, the physical properties (viscosity, surface tension, density, speed of sound, and bulk modulus) are evaluated at the initial bulk system temperature using values from the IAPWS R6-95(2018) formulation [15].

\section{APPENDIX B: TRANSITION ZONE SCALING ANALYSIS}

As shown in Fig. 2 of the main text, three regimes emerge when evaluating the dependence of the maximum pressure generated during cavity collapse on the degree of confinement. In the isochoric limit at sufficiently small confinement volumes, no significant pressure excursions are experienced. Conversely, at large confinement volumes, an isobaric limit is reached in which the bubble dynamics are unaffected by the rigid confinement and do not differ from an unconfined system. Between these two limits lies a transition zone that spans a few orders of magnitude in volume, in which the bubble dynamics begin to be affected by the rigid wall causing the peak collapse pressure to be suppressed.

Since the peak collapse pressure is integrally related to the maximum radius reached by the bubble during the growth period, the origin and behavior of the observed trends can be probed by performing a static force balance on the bubble at its point of maximum growth. During the tension phase of the ultrasonic stimulation, the ultrasonic pressure and pressure within the bubble [Eqs. (A16), (A17)] are balanced by the pressure due to the compression of the water volume [Eq. (A19)]. Taking the ultrasonic pressure to be equal to the driving pressure amplitude, the balance can be written as

$$
\begin{aligned}
K & \ln \left(\frac{R_{c}^{3}-R_{b, 0}^{3}}{R_{c}^{3}-R_{b, \max }^{3}}\right)+p_{l, 0} \\
& =\left(p_{l, 0}+\frac{2 \sigma}{R_{b, 0}}\right)\left(\frac{R_{b, 0}}{R_{b, \max }}\right)^{3 \gamma}-\frac{2 \sigma}{R_{b, \max }}+P_{A} .
\end{aligned}
$$

Shown in Fig. 6 is the normalized maximum bubble radius as a function of confinement radius for a range of values of liquid compressibility, $K^{-1}$, surface tension, $\sigma$, and initial bubble radius, $R_{b, 0}$. The location of the transition zone is found to be dependent on the compressibility $\left(K^{-1}\right)$, while the width of the transition zone is largely dependent on the surface tension $(\sigma)$. Although this static scaling analysis does not include the effect of viscosity or sonic velocity, detailed analysis utilizing the full bubble dynamics model shows that viscosity has a similar effect as surface tension, although to a weaker degree, and that sonic velocity has a similar effect to compressibility, which is expected due to their direct relationship [Eq. (A11)]. The initial bubble radius $\left(R_{b, 0}\right)$ is also found to affect the transition zone: smaller bubbles experience the effect of confinement at larger relative container volumes. This may be attributed to the increased significance of surface tension, which scales with the surface area to volume ratio and is thus more dominant in smaller bubbles. This relationship can be observed in Figs. 6(b) and 6(c). As the initial bubble size increases, this effect diminishes, and the relative critical confinement volume becomes independent of the initial bubble radius.

The scaling relation given in Eq. (B1) can be used to easily probe the discrete confinement volumes at which confinement
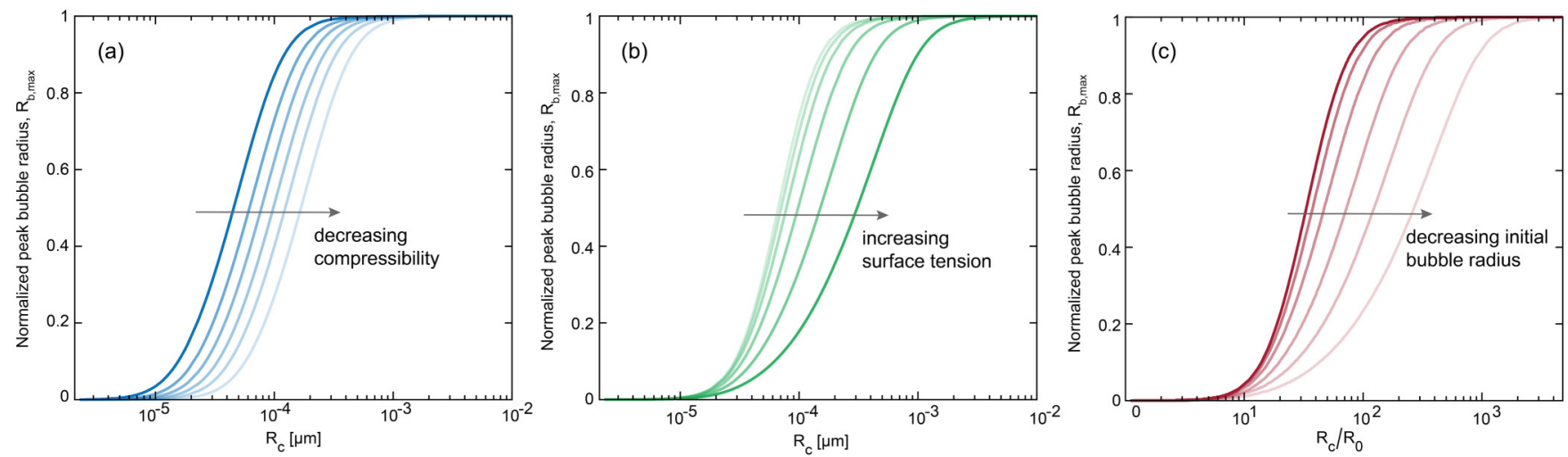

FIG. 6. Dependence of transition zone on system parameters: (a) compressibility, $K^{-1}$; (b) surface tension, $\sigma$; (c) initial bubble radius, $R_{b, 0}$. 
effects will be seen for arbitrary liquid materials with known compressibilities and surface tensions.

\section{APPENDIX C: NUCLEATION PARAMETERS}

Provided in this appendix is a very brief explanation of the parameters used to calculate the induction time for ice VII via classical nucleation theory. The approach is identical to that applied by Myint et al. [11], who followed the initial derivations by Kashchiev [21], and more information can be found in those works.

The thermodynamic driving force for nucleation is the difference in the bulk chemical potential between the solid and liquid phases:

$$
\Delta \mu=\mu_{\text {solid }}-\mu_{\text {liquid }} .
$$

We obtained temperature- and pressure-dependent chemical potential values for liquid water and ice VII from the equations of state developed by Myint et al. [20]. This chemical potential difference $\Delta \mu$, which scales with volume and drives ice formation, is then compared to the interfacial free energy $\sigma$, which scales with surface area and resists ice formation, to identify the critical radius at which an incipient ice cluster will form a stable nucleus. This critical cluster radius is defined as

$$
R^{*}=\frac{2 \sigma}{\rho_{\text {solid }} \Delta \mu},
$$

in which $\rho_{\text {solid }}$ is the density of the ice VII phase. The value of the interfacial free energy at a given cavitation pressure was calculated from a linear interpolation between the two reference points determined in Myint et al. [12], $\sigma=23.0 \mathrm{~mJ} / \mathrm{m}^{2}$ at $1.58 \mathrm{GPa}$ and $\sigma=129 \frac{\mathrm{mJ}}{\mathrm{m}^{2}}$ at $7 \mathrm{GPa}$.

The critical cluster radius is then employed in the definition of several other terms describing the size and behavior of a critical cluster, including:

The critical energy barrier to nucleation

$$
\Delta G^{*}=\frac{16 \pi \sigma^{3}}{3 \rho_{\text {solid }}^{2} \Delta \mu^{2}} .
$$

The critical cluster size

$$
n^{*}=\frac{32 \pi \sigma^{3}}{3 \rho_{\text {solid }}^{2} \Delta \mu^{3}} .
$$

The Zel'dovich factor, or the probability that a critical cluster will continue to grow, in which $T$ is the temperature and $k_{B}$ is the Boltzmann constant

$$
Z=\left(\frac{\Delta G^{*}}{3 \pi k_{B} T n^{*^{2}}}\right)^{1 / 2} .
$$

The growth rate of the stable critical cluster

$$
\gamma=\left(\frac{k_{B} T}{m}\right)^{1 / 2} \frac{\Delta \mu}{k_{B} T} .
$$

A frequency factor describing the attachment rate of new molecules to critical clusters, in which $V_{\text {solid }}$ is the molecular volume of the ice VII phase

$$
D^{*}=\frac{4 \pi R^{*^{2}} \gamma}{V_{\text {solid }}},
$$

and the curvature at the top of the energy barrier

$$
\lambda=2 \pi k_{B} T Z^{2} .
$$

Calculation of each of these parameters at a given temperature and pressure, starting from the bulk chemical potential difference, enables calculation of Eq. (3) in the main text, the induction time of a stable ice VII nucleus:

$$
\tau=\frac{8 k_{B} T}{\pi^{2} \lambda D^{*}} .
$$

It should be noted that no arbitrary scaling factor has been applied to this induction time, as was done by Myint et al. [11]. This results in our induction time calculation representing a conservative limit on the induction time of ice VII nucleation during cavity collapse (e.g., it provides the fastest possible induction time).
[1] E. J. Lavernia and T. S. Srivatsan, J. Mater. Sci. 45, 287 (2010).

[2] G. G. Stonehouse and J. A. Evans, J. Food Eng. 148, 74 (2015).

[3] R. J. de Vries, S. N. Tessier, P. D. Banik, S. Nagpal, S. E. J. Cronin, S. Ozer, E. O. A. Hafiz, T. M. van Gulik, M. L. Yarmush, J. F. Markmann, M. Toner, H. Yeh, and K. Uygun, Nat. Biotechnol. 37, 1131 (2019).

[4] T. A. Berendsen, B. G. Bruinsma, C. F. Puts, N. Saeidi, O. B. Usta, B. E. Uygun, M. L. Izamis, M. Toner, M. L. Yarmush, and K. Uygun, Nat. Med. 20, 790 (2014).

[5] H. Huang, M. L. Yarmush, and O. B. Usta, Nat. Commun. 9, 3201 (2018).

[6] B. J. Fuller, A. Petrenko, and E. Guibert, Nat. Biotechnol. 37, 1127 (2019).

[7] R. Hickling, Nature 206, 915 (1965).

[8] R. Hickling, Phys. Rev. Lett. 73, 2853 (1994).

[9] M. D. Luque de Castro and F. Priego-Capote, Ultrason. Sonochem. 14, 717 (2007).
[10] S. W. Young and W. J. Van Sicklen, J. Am. Chem. Soc. 35, 1067 (1913).

[11] P. C. Myint and J. L. Belof, J. Phys.: Condens. Matter 30, 233002 (2018).

[12] P. C. Myint, A. A. Chernov, B. Sadigh, L. X. Benedict, B. M. Hall, S. Hamel, and J. L. Belof, Phys. Rev. Lett. 121, 155701 (2018).

[13] M. J. Powell-Palm, A. Koh-Bell, and B. Rubinsky, Appl. Phys. Lett. 116, 123702 (2020).

[14] F. R. Gilmore, The Growth or Collapse of a Spherical Bubble in a Viscous Compressible Liquid (California Institute of Technology, California, 1952).

[15] International Association for the Properties of Water and Steam, IAPWS R6-95(2018) (Prague, Czech Republic, 2018).

[16] International Association for the Properties of Water and Steam, IAPWS R14-08(2011) (Plzeň, Czech Republic, 2011).

[17] G. W. Lee, W. J. Evans, and C.-S. Yoo, Phys. Rev. B 74, 134112 (2006). 
[18] A. E. Gleason, C. A. Bolme, E. Galtier, H. J. Lee, E. Granados, D. H. Dolan, C. T. Seagle, T. Ao, S. Ali, A. Lazicki, D. Swift, P. Celliers, and W. L. Mao, Phys. Rev. Lett. 119, 025701 (2017).

[19] D. M. Sterbentz, P. C. Myint, J. P. Delplanque, and J. L. Belof, J. Chem. Phys. 151, 164501 (2019).

[20] P. C. Myint, L. X. Benedict, and J. L. Belof, J. Chem. Phys. 147, 084505 (2017).
[21] D. Kashchiev, Surf. Sci. 14, 209 (1969).

[22] K. Kerboua and O. Hamdaoui, J. Acoust. Soc. Am. 146, 2240 (2019).

[23] M. J. Powell-Palm, B. Rubinsky, and W. Sun, Commun. Phys. 3, 39 (2020).

[24] B. Rubinsky, P. A. Perez, and M. E. Carlson, Cryobiology, 50, 121 (2005).

[25] H. G. Flynn, Cit. J. Acoust. Soc. Am. 57, 1379 (1975).

[26] R. Hicking and M. S. Plesset, Phys. Fluids 7, 7 (1964). 